\title{
A Case of Three Deltas in a Fingerprint
}

\author{
Singh RK ${ }^{1}$, Sharma $\mathbf{M}^{1^{*}}$, Tarannum $\mathbf{A}^{1}$, Pet-Paul $\mathbf{W}^{2}$, Bernard $L^{3}$
}

${ }^{1}$ Sherlock Institute of Forensic Science, Delhi, India

${ }^{2}$ Kwame Nkrumah University of Science and Technology, Ghana, West Africa

${ }^{3}$ Leddy Consultancy Ltd, Lismore, Ireland

*Corresponding author: Sharma M, Sherlock Institute of Forensic Science India, Delhi, India, Tel: 09630800789, E-mail: drsharmamahesh@gmail.com

Received date: December 01, 2017; Accepted date: March 23, 2018; Published date: March 28, 2018

Copyright: (c) 2018 Singh RK, et al. This is an open-access article distributed under the terms of the Creative Commons Attribution License, which permits unrestricted use, distribution, and reproduction in any medium, provided the original author and source are credited.

\begin{abstract}
Fingerprints begin forming at about the $6^{\text {th }}$ to the $13^{\text {th }}$ week of gestation. A number of factors determine the print pattern in the womb, including the density of the amniotic fluid surrounding the foetus, the size of the foetus, the friction in the womb, the location in the womb amongst others. Once developed the pattern remains constant throughout life. Basically there are three main patterns; The Loop, which rise up and loop back around, continuing on the same direction it started from and it accounts for about $70 \%$ of all fingerprints, Arches look like waves that rise and fall on the same fingerprint pattern, accounting for $5 \%$ whiles the Whorls appear as circular patterns accounting for $25 \%$. The loop has only one delta, the Arch no delta with the Whorl pattern having two deltas. In the case presented here, a young man of 30 years, who is physically fit and normal without any significant clinical condition apart from a developmental defect of the right thumb which makes it different from the left thumb. From the ten fingerprint patterns taken with the aid of a scanner from the subject, only the abnormal right thumb of the subject with a whorl fingerprint pattern has three deltas which are extremely uncommon and quite different to the traditional, widely known two deltas seen in all whorl patterns in fingerprints. Fingerprint deltas are only present in loops and whorls. There are basically four types of fingerprint whorls. The plain with two deltas, central pocket with two deltas, the double loop whorls with two deltas and accidental whorls which have two or more deltas. Theoretically, less than 1 in 1000 people has these extremely rare fingerprints. The three deltas in a fingerprint whorl pattern may exist in accidental whorls, but no mention is made of it in plain whorls which make this case perhaps the first ever of a known plain whorl with three deltas. This feature is very uncommon in normal patterns. In this case the thumb of this young man has developed abnormally in the womb and has a whorl pattern with three deltas. It is imperative this pattern is taken into consideration when evaluating or interpreting fingerprints. Though the number may be statistically small, it is still essential this is taken into account in manual and especially when using automatic fingerprint identification software to evaluate known and unknown fingerprints.
\end{abstract}

Keywords: Unconventional fingerprints; Three deltas fingerprints; Fingerprints analysis; Fingerprints whorl with three deltas

\section{Introduction}

Often an investigating officer discovers the single, partial, or only details of a fingerprint at a crime scene, which may be the only forensic evidence found at the scene. This single detail of a fingerprint helps the crime scene examiners in establishing the identity of an individual as the fingerprints of every individual are completely unique to that individual and no other living person. A fingerprint is the most reliable criteria for identification as they remain same for the entire duration of life. Fingerprinting is one of the oldest forensic evidence techniques which are used for the recognition and unique identification of an individual for law enforcement purposes [1]. An earthenware vessel estimated to be 6000 years old was discovered in northwest China and found to bear a clearly visible friction ridge impression and is considered to be the oldest friction ridge skin impression of a fingerprint found to date. It is still debateable whether they were placed by accident or specifically to make a decorative pattern on the pot [2]. In earlier times there was no opportunity for additional means of identification. Such evidence as signatures photographs and to personal effects could be used but in several cases of accident, murder, or suicide where the bodies of unknown were uncovered identification was quite problematic without fingerprints [3]. Fingerprint friction ridge skin has unique features that persist from birth until decomposition after death.

The unique fingerprint pattern begins with the formation by the sixth month of gestation. The uniqueness of friction ridge skin falls under the larger umbrella of biological uniqueness and no two points of reference of any living organism are exactly alike [4]. Around the $6^{\text {th }}$ and $7^{\text {th }}$ weeks of gestation, the paddle like hand of the foetus begins to develop fingers [5]. Volar pads starts appear on the palm (inter-digital pads first, followed by thenar and hypothenar pads) around $7^{\text {th }}$ to $8^{\text {th }}$ weeks of gestation and the major development of friction ridge skin occurs around $11^{\text {th }}$ and $20^{\text {th }}$ weeks of gestation [6]. The morphology of volar pads results into fingerprint patterns which are unique in terms of types and location of microscopic details [5]. According to Galton, fingerprints are classified into three features which are arches, loops and whorls. It is found that about $60-65 \%$ of population have loops, $30-35 \%$ of the population have whorls and $5-10 \%$ of population have arches [7]. Generally, Arches are formed when the ridges run from one side of the bulb to the other side of the bulb without forming any background turn or twist. Loops are formed when there is a single backward turn without any twist. Whorls are formed when there is at least one complete circle, they are also considered to include all duplex spirals [3]. Fingerprint deltas are only present in loops and whorls. There are basically four types of fingerprint whorls. The plain with two deltas, the central pocket with two deltas, the double loop whorls with two deltas and accidental whorls which have two or more deltas. The 
Page 2 of 4

whorl mainly comprises at least two deltas with a re-curve in front of each but there are a few accidental whorls which have three or more deltas characteristic of two or more different pattern types [8]. In the present study, a young man of 30 years, who is physically fit, had a developmental defect of the right thumb which makes it different from the left thumb. The right thumb which has developed abnormally in the womb has a whorl pattern with three deltas. Therefore, this unique pattern has been taken into consideration for the study and analysis of fingerprints.

\section{Materials and Methods}

In the present study, a unique fingerprint whorl pattern was discovered which shows the abnormal development of the subjects thumb during the fetal development of the person. This study should be taken into consideration for the improvement of the efficiency of biometric systems which have been in use for several years. Listed below are the materials used during this examination: Fingerprint Sample, Hand Lens, Scale, Scanner (cogent system) and Camera.

\section{Methodology}

To collect the fingerprint sample from the subject (age 30)who is identified as having this unique fingerprint pattern the subject was asked to clean his hands thoroughly with soap and water and dry them using towel to enhance getting both clear photographs and print of the fingerprint. Firstly photographs of the full hand were taken followed by a close up view photographs that focus on the thumbs so that the unique fingerprint pattern can be clearly visualized (Figure 1).

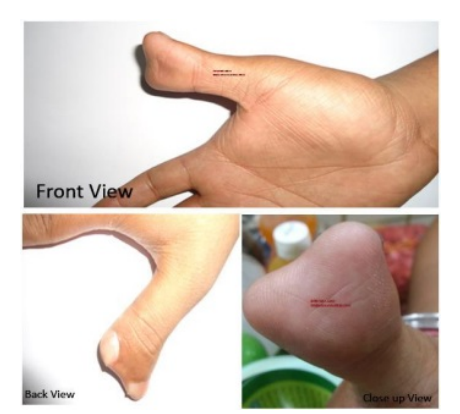

Figure 1: Front, back of hand and close up view of thumb.

For recording offline and online methods were used. In the offline method, the subject was asked to press his fingertip was pressed against an ink slab and then on the paper for getting the impression on the fingerprint card which was later on scanned for storing electronically. Care must be taken while recording the offline fingerprint it is important to prevent smudging of the print.

In the online method or live scan, the subject was asked to directly place the fingerprint over the scanner and record the fingerprint digitally. After acquiring fingerprints, details such as name, sex, and age, blood group were noted. After storing the fingerprint image electronically we proceeded to two levels of examination i.e. level 1 and level 2. The fingerprint pattern was studied with the help of magnifying glass for identifying the pattern and microscopic details in level 1 and level 2 respectively.

\section{Results}

The collected fingerprint patterns were examined for the two identifying patterns and microscopic features in level 1 and level 2 respectively. Level 1 mainly refers to the overall pattern formed by the flow papillary ridges on the papillary surface. These patterns are classified into generic classes by Galton et al $[3,9]$ into arches, loops, and whorls [6].

Whorls are further classified into four distinct group such as plain, central pocket loop, double loop, and accidental. Any whorl classified under accidental whorl classification will either contain two or more patterns [7]. The accidental whorl pattern may have three or more deltas with all the characteristics of two or more patterns types [8]. As per the theory concerning the whorl pattern, a whorl pattern with three deltas was recognized for the first time in the example shown in Figure 2 which was due to abnormal fetal development of the thumb.

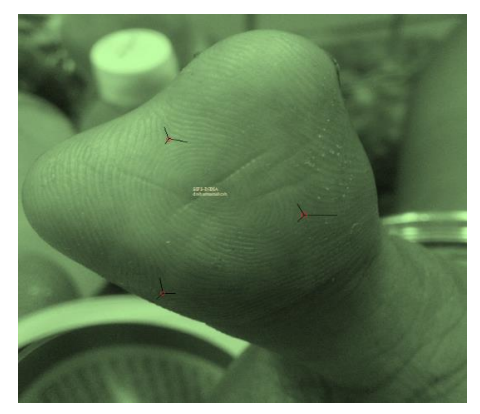

Figure 2: Whorl pattern showing three deltas.

After recognizing the pattern of the fingerprint, we proceeded for the level 2 examination. In level 2 mainly major ridge path deviations or micro features or Galton characteristics are observed and recognized.

The micro features observed in level two are individual to every person and even micro features are considered as individual characteristics also. The subjects fingerprints were taken for level 2 examination in which following micro features like core, delta, bifurcation, lake, hook/spur, bridge, enclosure, island, ridge ending and double bifurcation were observed shown in (Figure 3).

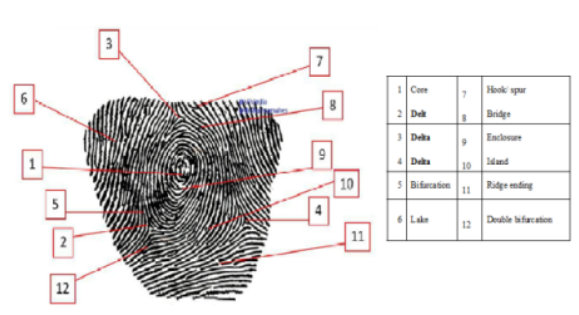

Figure 3: Minutiae's observed in the recorded fingerprint.

Below is the each micro features are described by the Figure 4 showing the micro features observed in the fingerprint pattern taken for study. 


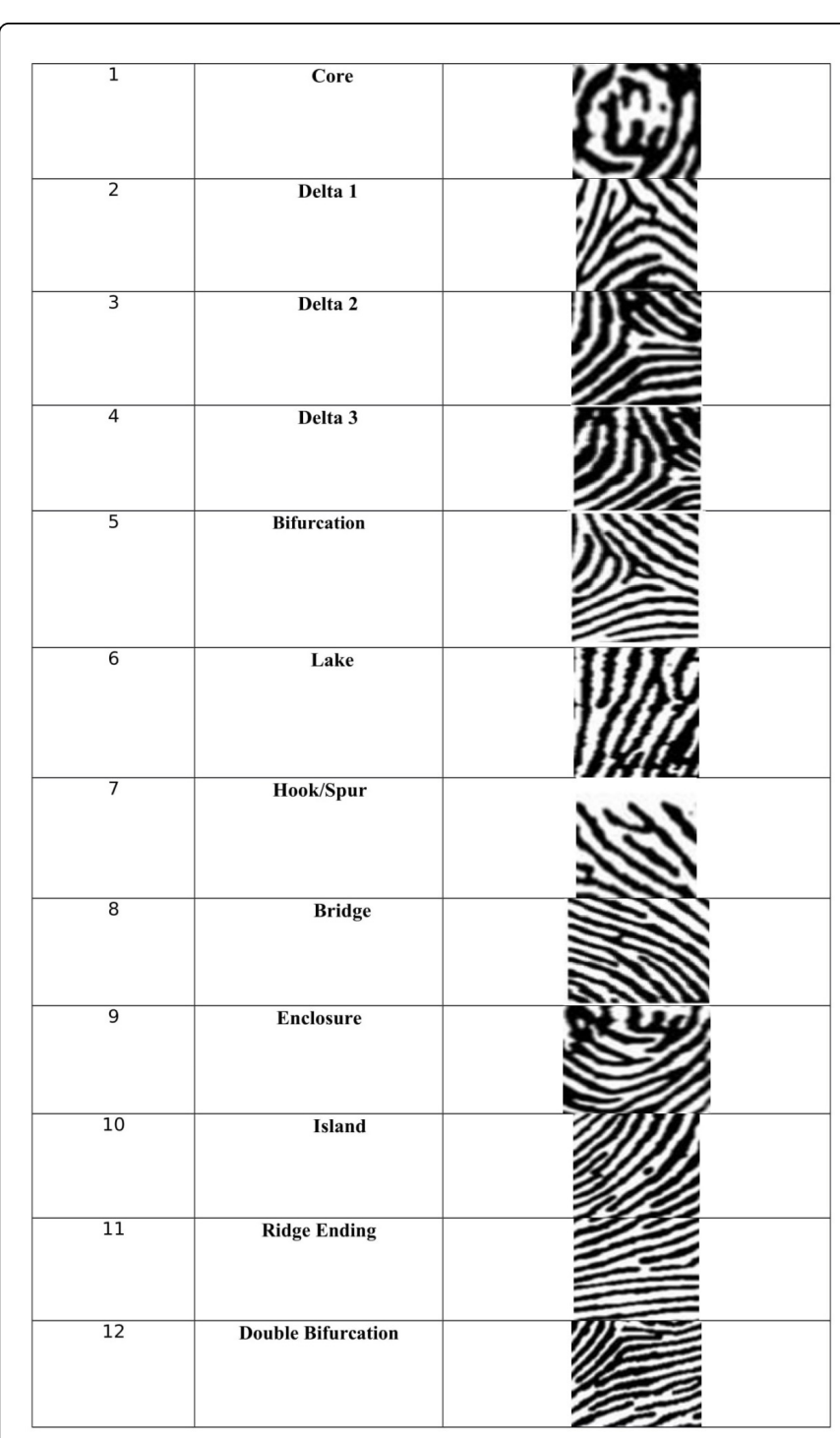

Figure 4: Showing the ridge characteristics observed in the fingerprint.

- Core: Center of the fingerprint pattern

- Delta: Point on a ridge at or in front of and nearest the center of the divergence of the type lines

- Bifurcation: Point where one friction ridge divides into two friction ridges.

- Lake: Large size enclosures are called in which single friction ridge first bifurcates and then converge and continues as a single friction ridge.

- Hook/Spur: Point where a ridge bifurcates with one short ridge branching from a long ridge.

- Bridge: Connecting friction ridge between the two parallel ridges which meets the ridge at an acute angle.

- Enclosure: Single friction ridge in the shape of ellipse that bifurcates to fuse and re-joins or converges and continues as a single friction ridge.

- Island: Ridges slightly longer than dots, occupying space between two temporarily divergent ridges
- Ridge Ending: Single friction ridge that terminates and come to an abrupt end within the friction ridge structure.

- Double Bifurcation: Point where ridge bifurcates again from a bifurcated ridge [5,10-15].

\section{Discussion}

Fingerprints are most commonly encountered as latent prints at the various crime scenes. In the present study, a unique fingerprint was observed for identifying the pattern and minutiae's or ridge characteristics. Galton [3], had already classified the fingerprint into three arches, loops and whorls on the basis of the degree of curvature of the ridges and even said that, "There are few ambiguous patterns, and others which are non-descript, but the former are uncommon and latter rare; As these exceptions give little real inconvenience in recognition".

Henry [9], classified the fingerprint pattern in four main types such as Arches, Loops, Whorls and Composite which are further classified into simple and tented arch, ulnar and radial loop, single and double whorls and central pockets loop, lateral pockets loop, twinned loop and accidental loops (irregular in outline to be grouped under any of the mentioned pattern). Later on (mentioned in his book "Friction ridge skin: Comparison and identification of fingerprints" that the accidental whorl patterns have three or more deltas, and have all the characteristics of two or more different pattern types. As per the present theory about the accidental whorl pattern with three or more delta, in this paper a similar type of fingerprint pattern is discussed for the first time. In this study, a young man fingerprint pattern is discussed who has a developmental defect in the right thumb.

\section{Conclusion}

In the present study, a young man of 30 years who has a developmental defect in the right thumb in comparison to his left thumb was observed. In this it was noted that the subject has an accidental whorl fingerprint pattern with three deltas which was identified as an unusual pattern in comparison to the broadly known whorl fingerprint pattern with similar type of minutiae's or ridge characteristics.

\section{References}

1. James SH, Jon JN (2003) Forensic science an introduction to scientific and investigative techniques. Boca Raton, CRC Press, USA.

2. The Fingerprint Sourcebook (2011) Washington DC: US Department of Justice, Office of justice programs, National Institute of Justice.

3. Galton F (1892) Fingerprints. London, New York: Macmillan and Co.

4. Diego-Alvarado DA, Ramírez AMM (2011) A biochemical hypothesis on the formation of fingerprints using a turing patterns approach. Theor Bio Med Model 8: 1-24.

5. Daluz HM (2014) Fundamentals of fingerprint analysis. Boca Raton, CRC Press, USA.

6. Christophe C, Lennard C, Margot P, Stoilovic M (2004) Fingerprints and other ridge skin impressions. Boca Raton, CRC Press, USA.

7. Saferstein R (2013) Forensic science: From the crime scene to the crime lab. 2nd edn, Upper Saddle River: Pearson.

8. Cowger JF (1992) Friction ridge skin: Comparison and identification of fingerprints. Boca Raton: CRC Press, USA.

9. Faulds H (1905) Guide to fingerprint identification. Park Street: Hanley.

10. https://www.gov.uk/government/uploads/system/uploads/ attachment_data/file/267523/FingerprintTerminology.pdf 
Citation: $\quad$ Singh RK, Sharma M, Tarannum A, Pet-Paul W, Bernard L (2018) A Case of Three Deltas in a Fingerprint. J Forensic Res 9: 417. doi: 10.4172/2157-7145.1000417

Page 4 of 4

11. Newman R (2010) Security and access control using biometric technologies. Boston, Mass: Course Technology.

12. Girard J (2008) Criminalistics: Forensic science and crime. Sudbury, MA: Jones and Bartlett Publishers, USA.

13. Nath S (2010) Fingerprint identification. New Delhi: Shiv Shakti Book Traders, Print, India.
14. Wang PSP (2011) Pattern recognition, machine intelligence and biometrics. Beijing: Higher Education Press.

15. Hawthorne MR (2009) Fingerprints: Analysis and understanding. Boca Raton: CRC Press. 